\title{
A novel schedule of accelerated partial breast radiation using intensity-modulated radiation therapy in elderly patients: survival and toxicity analysis of a prospective clinical trial
}

\author{
Mutlay Sayan, MD¹, Karen Wilson, BA, MEd, CCRP², Carl Nelson, MD¹, Havaleh Gagne, MD¹, \\ Deborah Rubin, MD', Ruth Heimann, MD, PhD' \\ 'Department of Radiation Oncology, University of Vermont Medical Center, Burlington, VT; \\ ${ }^{2}$ University of Vermont Cancer Center, Burlington, VT, USA
}

\begin{abstract}
Purpose: Several accelerated partial breast radiation (APBR) techniques have been investigated in patients with early-stage breast cancer (BC); however, the optimal treatment delivery techniques remain unclear. We evaluated the feasibility and toxicity of APBR delivered using intensity-modulated radiation therapy (IMRT) in elderly patients with stage I BC, using a novel fractionation schedule.

Materials and Methods: Forty-two patients aged $\geq 65$ years, with stage I BC who underwent breast conserving surgery were enrolled in a phase I/II study evaluating APBR using IMRT. Forty eligible patients received 40 Gy in 4 Gy daily fractions. Patients were assessed for treatment related toxicities, and cosmesis, before APBR, during, and after completion of the treatment.

Results: The median age was 73 years, median tumor size $0.8 \mathrm{~cm}$ and the median follow-up was 54 months. The 5 -year locoregional control was $97.5 \%$ and overall survival $90 \%$. Erythema and skin pigmentation was the most common acute adverse event, reported by 27 patients (69\%). Twenty-six patients (65\%) reported mild pain, rated 1-4/10. This improved at last follow-up to only $2(15 \%)$. Overall the patient and physician reported worst late toxicities were lower than the baseline and at last follow-up, patients and physicians rated cosmesis as excellent/good in $93 \%$ and $86 \%$, respectively.

Conclusion: In this prospective trial, we observed an excellent rate of tumor control with daily APBR. The acceptable toxicity profile and cosmetic results of this study support the use of IMRT planned APBR with daily schedule in elderly patients with early stage $B C$.
\end{abstract}

Keywords: Breast neoplasms, Intensity-modulated radiotherapy, Dose hypofractionation

\section{Introduction}

Breast conserving surgery (BCS) and whole breast radiation (WBR) for women with early-stage breast cancer has been shown to be an equivalent alternative to mastectomy for local control and survival. Although breast conservation therapy
(BCT) provides minimal long-term complications, favorable cosmetic outcomes and improved psychological well-being, not all women who are eligible are actually treated with BCT $[1,2]$. Furthermore, $15 \%-30 \%$ of patients treated with BCS do not receive adjuvant radiotherapy $[3,4]$. The underutilization of WBR has been associated with the length and frequency

Received 6 September 2016, Revised 19 October 2016, Accepted 11 November 2016.

Correspondence: Ruth Heimann, MD, PhD, University of Vermont Medical Center, 111 Colchester Avenue, Burlington, VT 05401, USA. Tel: +1-802-847-3506, Fax: +1-802-847-7413, E-mail: Ruth.Heimann@uvmhealth.org

(C) This is an Open Access article distributed under the terms of the Creative Commons Attribution Non-Commercial License (http://creativecommons.org/ licenses/by-nc/4.0/) which permits unrestricted non-commercial use, distribution, and reproduction in any medium, provided the original work is properly cited.

www.e-roj.org 
of treatment, which is 5 days a week for 6-7 weeks. This prolonged duration presents obstacles particularly for elderly patients and those who reside long distance from treatment facilities. Recently, accelerated partial breast radiation (APBR) was proposed as an alternative to WBR for patients with early stage breast cancer as it reduces treatment time.

The rationale for irradiating only a partial breast volume originates from studies reporting that 44\%-86\% of ipsilateral breast tumor recurrences (IBTR) develop at or near the tumor bed $[5,6]$. Therefore, it has been hypothesized that irradiating a smaller breast volume may be as efficacious as WBR, and, if less tissue is radiated, a shorter course of irradiation may potentially be offered using larger fraction sizes. However the larger daily doses may result in increased side effect and treating only part of the breast tissue in higher recurrent rate.

Several techniques for APBR have been reported including multicatheter brachytherapy [7], MammoSite brachytherapy [8] and external beam radiation therapy (EBRT) with 3-dimensional conformal radiation therapy (3D-CRT) which is the most commonly used method in the National Surgical Adjuvant Breast and Bowel Project (NSABP) B-39/RTOG 0413 trial or intensity-modulated radiation therapy (IMRT) $[9,10]$. External beam approach with 3D-CRT offers superior dose homogeneity compared to invasive techniques; however, the disadvantage of 3D-CRT is irradiation of a larger volume of the ipsilateral breast, as well as more dose to heart, and lung tissue [11]. The currently published studies on APBR are bid fractionation for a week. For patients who travel long distances for therapy a bid schedule is difficult. Therefore we tested a daily fractionation scheme for a total of 10 fractions 5 days a weak using IMRT. IMRT improves conformal dose coverage by using inverse planning algorithms to deliver an optimal dose to the target volume [12] and provides better normal tissue sparing compared to 3D-CRT $[13,14]$. It allows control of the dose in the untreated part of the breast which is important if retreatment is necessary at the time of a recurrence. There are only a limited number of studies using IMRT.

In this phase I/II single arm study, we evaluated the feasibility and toxicity of APBR delivered using IMRT to the lumpectomy cavity of postmenopausal patients with stage I breast cancer in an accelerated daily fractionation schedule.

\section{Materials and Methods}

\section{Study population}

Forty-two patients with stage I breast cancer were enrolled in an Institutional Review Board approved phase I/II feasibility study at the University of Vermont Medical Center between June 2006 and May 2013. The study was conducted in accordance with the principles of the Declaration of Helsinki and all patients provided written informed consent.

Eligibility criteria included: (1) female patient with invasive ductal carcinoma (IDC) American Joint Committee on Cancer (AJCC) stage I, post-partial mastectomy and axillary node dissection or sentinel lymph node biopsy; (2) age $\geq 65$ years; (3) visible lumpectomy cavity on planning computed tomography (CT); (4) ability to begin radiation treatment 3-8 weeks postsurgery, unless receiving chemotherapy first; and (5) if chemotherapy was planned, radiation treatment would start 3-5 weeks after completion of chemotherapy. Patients were not eligible if there was lymphovascular invasion or if the surgical margins were positive. Hormonal therapy was allowed; however, it was preferable to begin hormonal therapy after completion of radiotherapy.

\section{Simulation and treatment technique}

A CT simulations was performed with the patient lying supine with both arms raised above the head on a breast board with Vac-Lok immobilization (CIVCO Radiotherapy, Orange City, IA, USA). Based on the planning CT scan, the lumpectomy cavity was outlined by the treating radiation oncologist. Both breasts, both lungs, and the heart were also contoured either by a dosimetrist or the treating physician. The clinical target volume (CTV) was defined as the excision cavity $+1.0 \mathrm{~cm}(5 \mathrm{~mm}$ from skin surface and from pectoralis muscle). The planning target volume (PTV) was a $0.5 \mathrm{~cm}$ expansion around the CTV. The final PTV was modified to exclude the first $5 \mathrm{~mm}$ of tissue from the skin surface and the portion extending into the lung.

IMRT was used for treatment planning using Pinnacle planning system (Philips Medical System, Cleveland, $\mathrm{OH}$, USA). Planning guidelines were to cover the PTV with at least $95 \%$ isodose line. Normal tissue guidelines were such that (1) for the remainder of the breast excluding the PTV $<50 \%$ of the volume was to receive $\geq 50 \%$ of the dose and $<25 \%$ to receive the prescription dose; (2) the contralateral breast to receive $<3 \%$ of the prescribed dose; $(3)<15 \%$ of the ipsilateral lung to receive $30 \%$ of the prescribed dose and $<10 \%$ of the contralateral lung to receive 5\% the dose; (4) depending on the laterality, $<40 \%$ of the heart to receive $5 \%$ of the prescribed dose; and (5) for left sided breast cancer an $<5 \%$ of the heart to receive $\geq 5 \%$ of dose. Ten fractions of $4 \mathrm{~Gy}$ were delivered once daily 5 days a week for a total dose of $40 \mathrm{~Gy}$. A representative plan is shown in Fig. 1.

The patients were seen weekly while on treatment and 


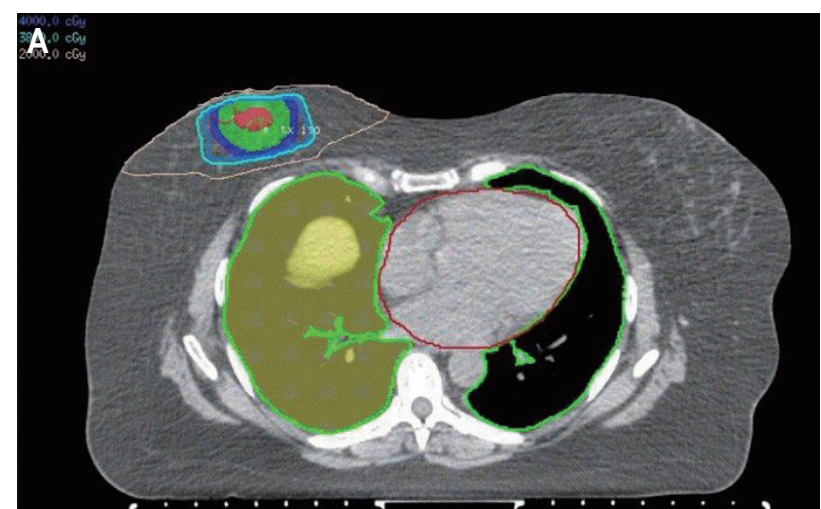

B

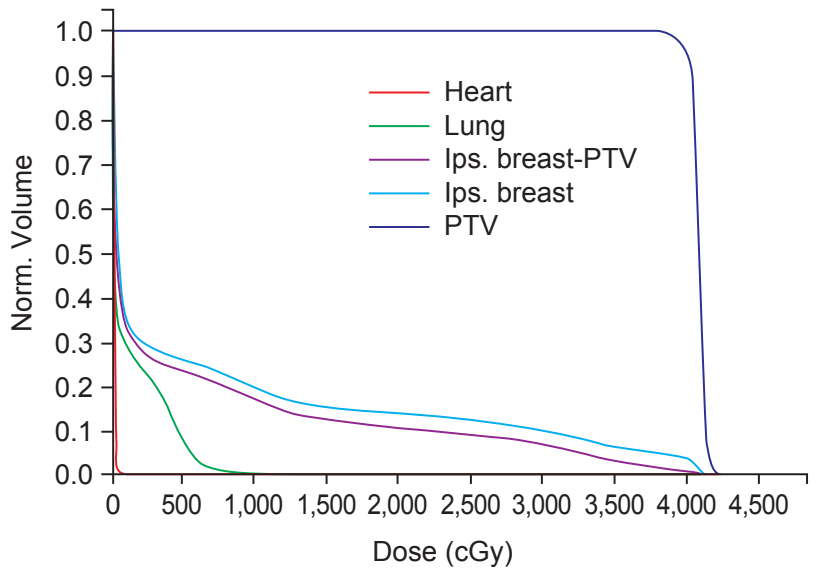

Fig. 1. An example of (A) axial view of planning computed tomography image and (B) dose volume histogram for accelerated partial breast radiation. PTV, planning target volume; Ips., ipsilateral; Norm., normal.

for additional 4 weeks by the treating radiation oncologist. Subsequent radiation oncology follow ups were at 6 months, 1 year and yearly after for 5 years.

\section{Toxicity assessment}

RTOG/EORTC toxicity grading was used in this study. Patient reported acute toxicities included those that occurred during radiotherapy and up to 4 weeks after completion of treatment. Infection and healing problems were also recorded. Chronic toxicities and cosmetic outcomes were evaluated by both the treating physician and the patients at 6 months and yearly after for 5 years. Patients were asked to describe late toxicities using a four-point scale $(0$, none; 1 , slight; 2 , moderate; 3 , noticeable). Treating physicians graded toxicities also using a similar scale ( 0 , none; 1 , mild; 2 , moderate; 3 , severe). The first cosmetic assessment was done at baseline prior to the start of radiation therapy. The overall cosmetic assessment was done using a modified Harvard criteria for cosmesis [15] where a score of 9 or 10 (excellent) was given if the treated breast looks exactly the same as the opposite breast, 7-8 (good) minimal but identifiable effects of radiation; 4-6 (fair) significant effects of radiation on the breast; 1-3 (poor) severe normal tissue sequelae.

\section{Statistical analysis}

Statistical analyses were performed with the SPSS statistical software ver. 17.0 (SPSS Inc., Chicago, IL, USA). Descriptive statistics were compiled to characterize the patient populations. Toxicities and cosmesis were compared across the variables of interest using either chi-squared tests or ANOVA. Significance was assessed at 0.05 .

\section{Results}

\section{Patient characteristics}

Forty-two patients were enrolled in this study from June 2006 to May 2013, however one patient withdraw from the study and another patient was determined to be not eligible. The patient characteristics for the enrolled 40 patients are shown in Table 1. Twenty-one patients (52.5\%) presented with a

Table 1. Characteristics of 40 enrolled patients

\begin{tabular}{lc}
\hline \multicolumn{1}{c}{ Characteristic } & Value \\
\hline Age $(\mathrm{yr})$ & $73(65-88)$ \\
Histology & \\
IDC & $36(90)$ \\
Other & $4(10)$ \\
Tumor size $(\mathrm{cm})$ & $0.8(0.3-1.7)$ \\
Margin size $(\mathrm{cm})$ & $0.5(0.06-1)$ \\
No. of nodes sampled & $2(1-13)$ \\
T Stage & \\
T1a & $9(22.5)$ \\
T1b & $19(47.5)$ \\
T1c & $12(30)$ \\
Grade & \\
G1 & $20(50)$ \\
G2 & $16(40)$ \\
G3 & $4(10)$ \\
ER status & \\
Positive & $37(92.5)$ \\
Negative & $3(7.5)$ \\
PR status & \\
Positive & $33(82.5)$ \\
Negative & $7(17.5)$ \\
\hline
\end{tabular}

Values are presented as median (range) or number (\%). IDC, invasive ductal carcinoma; ER, estrogen receptor; PR, progesterone receptor. 
Table 2. Patient reported acute radiation toxicities $(n=39)$

\begin{tabular}{lc}
\hline Adverse event & $\begin{array}{c}\text { No. of patients with } \\
\text { acute toxicity (\%) }\end{array}$ \\
\hline Skin toxicity & \\
Erythema/pigmentation & $27(69)$ \\
Dry desquamation & $3(7.7)$ \\
Edema & $24(61.5)$ \\
Subcutaneous toxicity & $20(51)$ \\
Pain & \\
0 & $11(27.5)$ \\
$1-4$ & $26(65)$ \\
$\geq 5$ & $3(7.5)$ \\
\hline
\end{tabular}

Table 3. Patient and physician reported baseline and late toxicities

\begin{tabular}{lcc}
\hline & Baseline & Late $^{\text {a) }}$ \\
\hline Patient reports $(n=39)$ & & \\
Pigmentation/dry desquamation & $15(38.5)$ & $9(23)$ \\
Breast edema & $18(46)$ & $3(8)$ \\
Subcutaneous toxicity & $15(38.5)$ & $13(33)$ \\
Telangiectasia & $2(5)$ & $5(13)$ \\
Contour defect & $24(61.5)$ & $23(59)$ \\
Clothing restriction & $0(0)$ & $0(0)$ \\
Physician reports $(n=37)$ & $13(35)$ & $16(43)$ \\
Pigmentation & $19(51)$ & $11(30)$ \\
Breast edema & $12(32)$ & $26(70)$ \\
Subcutaneous toxicity & $3(8)$ & $7(19)$ \\
Telangiectasia & $17(46)$ & $31(84)$ \\
Volume loss & $16(43)$ & $19(51)$ \\
Retraction or contour defect &
\end{tabular}

Values are presented as number (\%).

${ }^{a}$ For each patient the worst toxicity was scored.

right-sided tumor and 19 (47.5\%) with a left-sided tumor. All patients had negative margins. Majority of the tumors (90\%) were grade 1 and 2 . All of the patients underwent axillary evaluation. The majority of the tumors were estrogen receptor (ER) and progesterone receptor (PR) positive. Adjuvant chemotherapy was not utilized in any patients. Adjuvant hormonal therapy was given at the discretion of treating medical oncologist.

\section{Treatment related toxicities}

Patient reported acute toxicities are shown in Table 2. Erythema and skin pigmentation was the most common adverse event, reported by 27 patients (69\%). Breast edema and subcutaneous toxicities consisting of skin thickening and hardening were reported by 24 patients (61.5\%) and 20 patients (51\%), respectively. Dry desquamation was reported by 3 patients (7.7\%). Majority of the patients reported mild pain, rating 1-4 out of 10 , during treatment. While 3 patients (7.5\%) reported grade $\geq 5$ pain, 11 patients (27.5\%) experienced no pain. No treatment related respiratory symptoms were reported. No infection or wound dehiscence were seen.

Patient and physician-reported late toxicities are shown in Table 3. Toxicity occurring at the 6 months follow up or later are included. Nine patients (23\%) reported changes in pigmentation and dry desquamation, 3 patients (8\%) late breast edema. Both were substantially lower than at baseline. Physicians assessed more women with late changes in pigmentation 16 (43\%) and breast edema 11 (30\%). Compared to baseline only telangiectasias were reported more frequently. The proportion of women reporting breast edema was low, however majority had contour defect.

Most toxicities were grades 1 and 2. Grade 3 edema, subcutaneous toxicity and moderate/severe contour defect were reported by 1 patient (3\%), 4 (10\%), 5 (13\%), respectively. More physicians than patients reported skin pigmentation changes, breast edema, and subcutaneous toxicity. Majority of the women had some volume loss compared to contralateral breast but none reported clothing restrictions.

The prospectively gathered treatment-related pain reported by the patients on a scale of 1-10 is shown in Table 4. And $67 \%-85 \%$ of the patients were free of breast or nipple pain and $86 \%-100 \%$ of the patients had no chest wall or axilla pain at the various follow-up times. Grade 1-4 breast or nipple pain was experienced by 8 patients (27\%) in the first year after completing radiation therapy; however, this improved by the last follow up to only 2 patients (15\%) reporting this level of pain. Grade $\geq 5$ pain in the breast or nipple was reported by 2 patients and by one patient in the chest wall and axilla. No grade $\geq 5$ pain was reported during the last follow up visits.

Overall, 87\%-93\% of patients scored their overall cosmetic result as 'excellent' or 'good' (Fig. 2). Similarly, treating physicians rated $86 \%-100 \%$ of patients' cosmesis as 'excellent' or 'good.' While physicians did not report any 'poor' outcomes, $3 \%-6 \%$ of the patients rated their cosmetic outcome as 'poor.' There was not deterioration in the overall cosmetic score with increase follow-up time.

\section{Outcomes}

The median follow-up time at the time of this analysis was 54 months. The 5-year locoregional control rate was 97.5\%. One patient with BRCA2 mutation had a recurrence in the same quadrant 20 months after radiation. Her disease was initially stage pT1bpNO poorly differentiated IDC, ER/PR positive, 
Table 4. Patient reported chronic pain

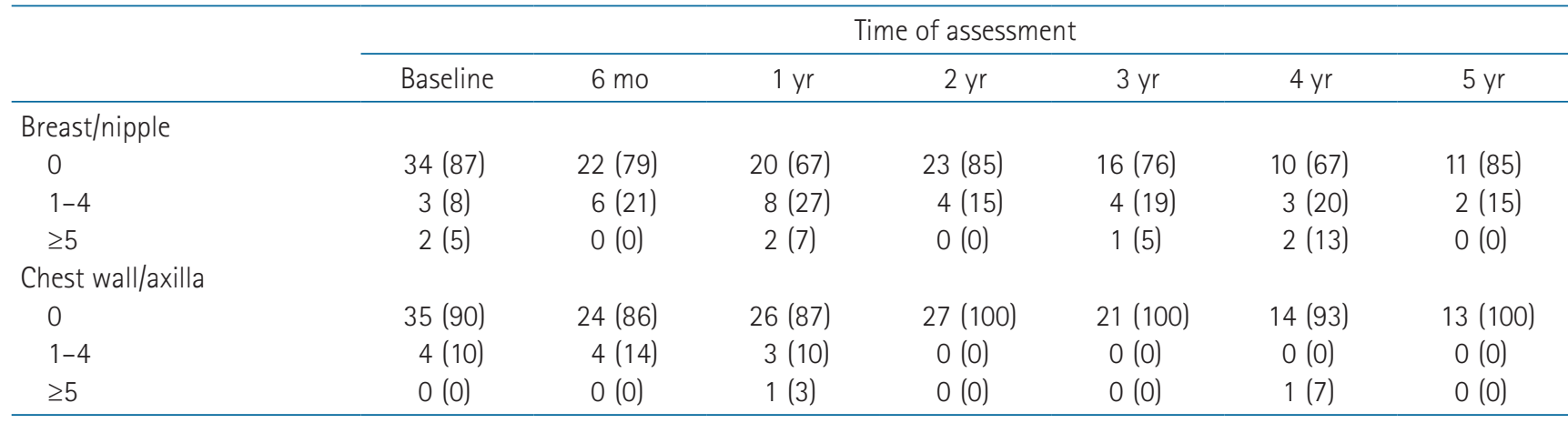

Values are presented as number (\%).

and HER-2 negative and the recurrence of disease was stage pT1bpNO moderately differentiated IDC, triple negative, which was treated with bilateral total mastectomy. The 5-year overall survival rate was 90\%. During the follow-up time period, 4 patients $(10 \%)$ died of non-breast cancer related causes without evidence of local recurrence.

\section{Discussion and Conclusion}

In this study, we have shown the feasibility, safety, and clinical outcomes of delivering APBR using IMRT. APBR is more convenient in comparison to whole breast irradiation (WBI) for women undergoing BCS, therefore a growing number of facilities offer this new treatment approach. However, as the results of ongoing randomized clinical trials are not available,

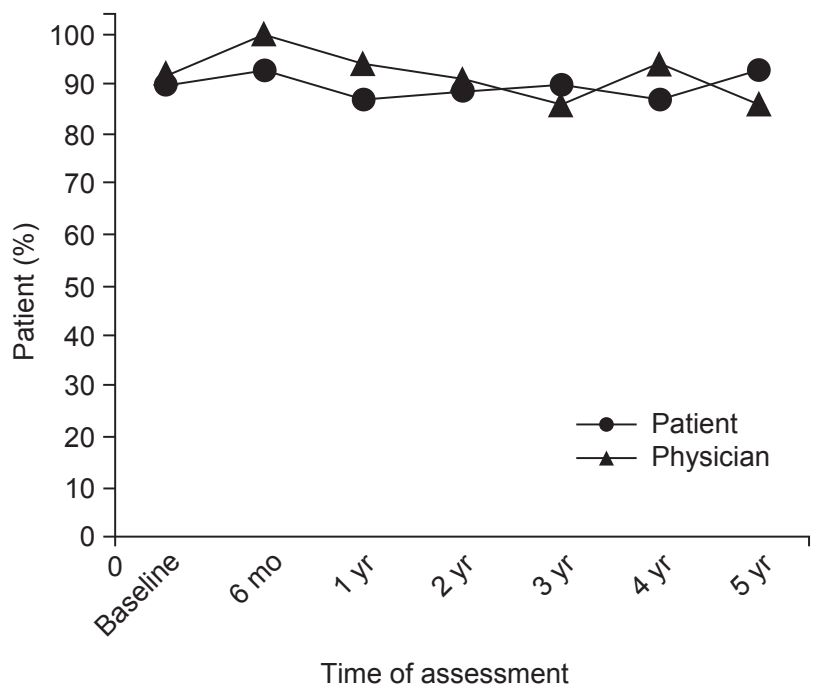

Fig. 2. Patient and physician reported excellent/good cosmetic outcomes. the long-term disease control, treatment related toxicity, cosmetic outcome, treatment schedule, and APBR delivery techniques remain controversial. In this prospective trial with a 54-month median follow-up, we demonstrate excellent tumor control with acceptable toxicity and cosmetic results. The unique aspects of our study include the elderly nature of our patients and daily fractionation scheme. The daily fractionation schedule logistically is easier for patients particularly elderly and if there is long distance travel involved.

Various APBR delivery techniques have been described in the past decade, including a small number of studies using IMRT $[16,17]$. These reports mostly used a bid schedule. Although the popularity of APBR with breast cancer has increased and new delivery techniques have emerged, a limited number of studies actually justify the chosen radiation dose schedule. In our study, we applied a daily fractionation scheme consisting of a total of 10 fractions 5 days a week using IMRT. Computation of biological equivalent dose (BED) can be used to quantify the expected biological effect associated with the treatment of a large fraction dose in a short period and compare these anticipated biological effects with commonly used WBR protocols. Similar to prior reports, we used the $\alpha / \beta$ value of $4 \mathrm{~Gy}$ to determine the tumor control BED and the $\alpha / \beta$ value of 2 and $10 \mathrm{~Gy}$ for the toxicities [18]. The tumor control BED value in our study was $80 \mathrm{~Gy}$, which was in the range of BED values (72-99 Gy) from different standard WBR protocols. Calculated toxicity BED values in our study were lower than the standard WBR protocols for $\alpha / \beta$ of 10 and slightly higher for $\alpha / \beta$ of 2 . These observations and previous reports guided us in determining the use of dose/fractionation schedule in our study.

The most common acute toxicity in our study was erythema and skin pigmentation (69\%) similar to previously reported studies in which $50 \%$ of patients with stage I/II breast cancer 
treated with APBR using IMRT, 38.5 Gy in 10 fractions BID, developed erythema and 33\% had hyperpigmentation [16]. In our cohort, breast edema was reported by $61 \%$ of the patients as an acute toxicity. However, during long term follow-up, only $8 \%$ of patients reported having breast edema. While physician reported higher rates of late breast edema (30\%), this was still comparable to other studies of APBR using EBRT that report breast edema in 12\%-29\% of patients $[16,17,19,20]$. In our cohort, highest grade subcutaneous toxicity occurred in $51 \%$ of patients as an acute toxicity. However, during the long term follow-up only 33\% of the patients reported having subcutaneous toxicity. Late subcutaneous toxicities reported by patients and treating physicians in our study were similar to previous studies using APBR using IMRT $[10,16]$ and 3D-CRT [19]. The overall proportion of late toxicities reported by patients with the exception of telangiectasia was lower than at baseline and also lower than physician reported late toxicities. It has been previously observed that physicians can be more critical in assessing cosmesis compared to patients.

At the time of treatment, 65\% of patients reported mild pain; however, this improved with time. Patient reported breast pain was modestly more prevalent at 1 year (27\%). This rate of mild breast pain is similar to the proportion reported in a trial of WBR (24\%) [21] and also in patients treated with APBR using 3D-CRT (26\%) [22]. In our study, only 15\% of patients reported mild pain at their 5-year follow up appointment, however $41 \%$ of patients treated with APBI using 3D-CRT reported pain at their 5 year appointment [19]. Furthermore, Lei et al. [17] reported that $26.3 \%$ of patients treated with APBI with IMRT, 38.5 Gy in 10 fraction BID, had pain at last follow-up. The lower proportion of pain during the follow-up period is most likely due to the dose fractionation scheme used in our study.

Cosmetic outcomes were evaluated by both treating physicians and patients in order to avoid observation bias. Over the follow-up period, 87\%-93\% of patients and 86\%-100\% of physicians scored the overall cosmesis as excellent or good. Twenty-one percent of patients treated with APBR using IMRT, $38.5 \mathrm{~Gy}$ in $3.85 \mathrm{~Gy}$ fractions twice daily, at the University of Michigan developed unacceptable adverse cosmesis, leading to early closure of the trial [23]. Excellent or good cosmesis was reported in $73 \%-82 \%$ of patients treated with APBR using 3D-CRT with various fractionation schedule $[19,22,24,25]$.

We can conclude that the fractionation scheme used in our study did not compromise cosmesis or outcomes. During the follow-up period there was a single patient with a recurrence in the same quadrant as the initial breast cancer. There were no axillary or systemic recurrences or breast cancer related deaths.

In conclusions, APBR is an evolving standard of care and the debate over the clinical role of different APBR delivery techniques will likely remain unresolved until large randomized clinical trials are designed to compare them. Nevertheless, equivalent survival outcomes and favorable toxicity profiles in our study support the use of APBR with IMRT with a daily 2 weeks schedule as a promising treatment option for elderly patients with early stage breast cancer.

\section{Conflict of Interest}

No potential conflict of interest relevant to this article was reported.

\section{References}

1. Morrow M, White J, Moughan J, et al. Factors predicting the use of breast-conserving therapy in stage I and II breast carcinoma. J Clin Oncol 2001;19:2254-62.

2. Acharya S, Hsieh S, Michalski JM, Shinohara ET, Perkins SM. Distance to radiation facility and treatment choice in earlystage breast cancer. Int J Radiat Oncol Biol Phys 2016;94:691-9.

3. Lazovich DA, White E, Thomas DB, Moe RE. Underutilization of breast-conserving surgery and radiation therapy among women with stage I or II breast cancer. JAMA 1991;266:3433-8.

4. Ballard-Barbash R, Potosky AL, Harlan LC, Nayfield SG, Kessler LG. Factors associated with surgical and radiation therapy for early stage breast cancer in older women. J Natl Cancer Inst 1996;88:716-26.

5. Malmstrom P, Holmberg L, Anderson $H$, et al. Breast conservation surgery, with and without radiotherapy, in women with lymph node-negative breast cancer: a randomised clinical trial in a population with access to public mammography screening. Eur J Cancer 2003;39:1690-7.

6. Clark RM, Whelan $T$, Levine $M$, et al. Randomized clinical trial of breast irradiation following lumpectomy and axillary dissection for node-negative breast cancer: an update: Ontario Clinical Oncology Group. J Natl Cancer Inst 1996;88:1659-64.

7. Arthur DW, Winter K, Kuske RR, et al. A Phase II trial of brachytherapy alone after lumpectomy for select breast cancer: tumor control and survival outcomes of RTOG 95-17. Int J Radiat Oncol Biol Phys 2008;72:467-73.

8. Cuttino LW, Keisch M, Jenrette JM, et al. Multi-institutional experience using the MammoSite radiation therapy system in the treatment of early-stage breast cancer: 2-year results. Int J Radiat Oncol Biol Phys 2008;71:107-14.

9. Vicini F, Winter K, Wong J, et al. Initial efficacy results of RTOG 0319: three-dimensional conformal radiation therapy (3D-CRT) 
confined to the region of the lumpectomy cavity for stage I/ II breast carcinoma. Int J Radiat Oncol Biol Phys 2010;77:1120-7.

10. Liss AL, Ben-David MA, Jagsi $R$, et al. Decline of cosmetic outcomes following accelerated partial breast irradiation using intensity modulated radiation therapy: results of a single-institution prospective clinical trial. Int J Radiat Oncol Biol Phys 2014;89:96-102.

11. Kim Y, Parda DS, Trombetta MG, et al. Dosimetric comparison of partial and whole breast external beam irradiation in the treatment of early stage breast cancer. Med Phys 2007;34:4640-8.

12. Oliver $M$, Chen J, Wong E, Van Dyk J, Perera F. A treatment planning study comparing whole breast radiation therapy against conformal, IMRT and tomotherapy for accelerated partial breast irradiation. Radiother Oncol 2007;82:317-23.

13. Rusthoven $K E$, Carter DL, Howell $K$, et al. Accelerated partialbreast intensity-modulated radiotherapy results in improved dose distribution when compared with three-dimensional treatment-planning techniques. Int J Radiat Oncol Biol Phys 2008;70:296-302.

14. Saibishkumar EP, MacKenzie MA, Severin D, et al. Skin-sparing radiation using intensity-modulated radiotherapy after conservative surgery in early-stage breast cancer: a planning study. Int J Radiat Oncol Biol Phys 2008;70:485-91.

15. Rose MA, Olivotto I, Cady $B$, et al. Conservative surgery and radiation therapy for early breast cancer: long-term cosmetic results. Arch Surg 1989;124:153-7.

16. Lewin AA, Derhagopian $R$, Saigal $K$, et al. Accelerated partial breast irradiation is safe and effective using intensitymodulated radiation therapy in selected early-stage breast cancer. Int J Radiat Oncol Biol Phys 2012;82:2104-10.

17. Lei RY, Leonard $C E$, Howell $K T$, et al. Four-year clinical update from a prospective trial of accelerated partial breast intensitymodulated radiotherapy (APBIMRT). Breast Cancer Res Treat 2013;140:119-33.

18. Williams MV, Denekamp J, Fowler JF. A review of alphal beta ratios for experimental tumors: implications for clinical studies of altered fractionation. Int J Radiat Oncol Biol Phys 1985;11:87-96.

19. Shah C, Wilkinson JB, Lanni T, et al. Five-year outcomes and toxicities using 3-dimensional conformal externa beam radiation therapy to deliver accelerated partial breast irradiation. Clin Breast Cancer 2013;13:206-11.

20. Formenti SC, Hsu H, Fenton-Kerimian M, et al. Prone accelerated partial breast irradiation after breast-conserving surgery: five-year results of 100 patients. Int J Radiat Oncol Biol Phys 2012;84:606-11.

21. Hopwood P, Haviland JS, Sumo G. Comparison of patientreported breast, arm, and shoulder symptoms and body image after radiotherapy for early breast cancer: 5-year follow-up in the randomised Standardisation of Breast Radiotherapy (START) trials. Lancet Oncol 2010;11:231-40.

22. Berrang TS, Olivotto I, Kim DH, et al. Three-year outcomes of a Canadian multicenter study of accelerated partial breast irradiation using conformal radiation therapy. Int J Radiat Oncol Biol Phys 2011;81:1220-7.

23. Jagsi R, Ben-David MA, Moran JM, et al. Unacceptable cosmesis in a protocol investigating intensity-modulated radiotherapy with active breathing control for accelerated partial-breast irradiation. Int J Radiat Oncol Biol Phys 2010;76:71-8.

24. Olivotto IA, Whelan TJ, Parpia S, et al. Interim cosmetic and toxicity results from RAPID: a randomized trial of accelerated partial breast irradiation using three-dimensional conformal external beam radiation therapy. J Clin Oncol 2013;31:403845.

25. Leonard KL, Hepel JT, Hiatt JR, Dipetrillo TA, Price LL, Wazer $D E$. The effect of dose-volume parameters and interfraction interval on cosmetic outcome and toxicity after 3-dimensional conformal accelerated partial breast irradiation. Int J Radiat Oncol Biol Phys 2013;85:623-9. 